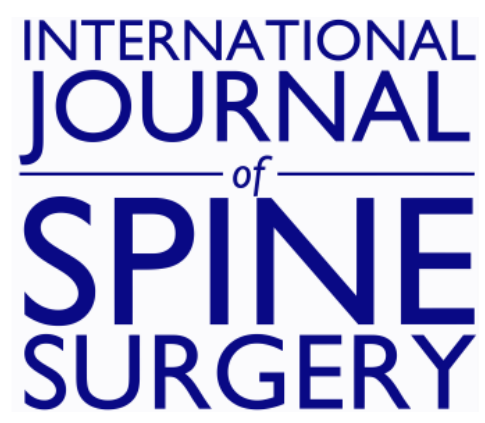

\title{
Spinal fusion with demineralized calf fetal growth plate as novel biomaterial in rat model: a preliminary study
}

Amin Bigham-Sadegh, Iraj Karimi, Ahmad Oryan, Elena Mahmoudi and Zahra Shafiei-Sarvestani

Int J Spine Surg 2014, 8 ()

doi: https://doi.org/10.14444/1005

http://ijssurgery.com/content/8/5

This information is current as of April 25, 2023.

Email Alerts Receive free email-alerts when new articles cite this article. Sign up at:

http://ijssurgery.com/alerts

The International Journal of Spine Surgery

2397 Waterbury Circle, Suite 1,

Aurora, IL 60504, Phone: +1-630-375-1432 


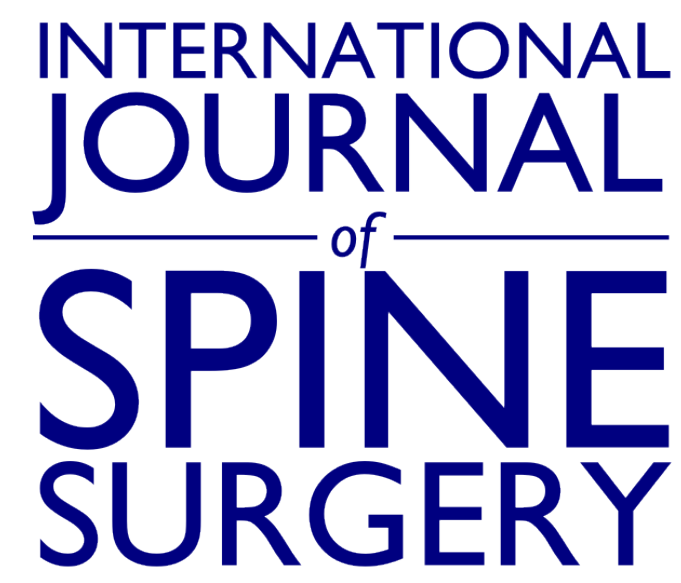

This article generously published free of charge by the International Society for the Advancement of Spine Surgery.

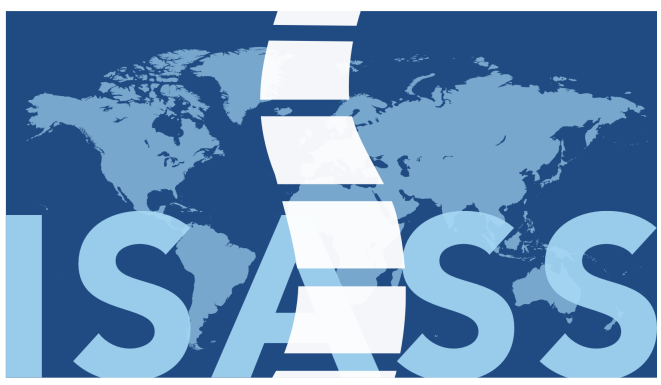

INTERNATIONAL SOCIETY for the ADVANCEMENT of SPINE SURGERY 


\section{Spinal fusion with demineralized calf fetal growth plate as novel biomaterial in rat model: a preliminary study}

Amin Bigham-Sadegh, DVM, Ph.D, ${ }^{1}$ Iraj Karimi, DVM, Ph.D, ${ }^{2}$ Ahmad Oryan, DVM, Ph.D, ${ }^{3}$ Elena Mahmoudi, DVM, ${ }^{4}$ Zahra Shafiei-Sarvestani, DVM, Ph.D ${ }^{1}$

${ }^{1}$ Department of Veterinary Surgery and Radiology, Faculty of Veterinary Medicine, Shahrekord University, Shahrekord, Iran ${ }^{2}$ Department of Veterinary Pathobiology, Faculty of Veterinary Medicine, Shahrekord University, Shahrekord, Iran ${ }^{3}$ Department of Veterinary Pathobiology, School of Veterinary Medicine, Shiraz University, Shiraz, Iran ${ }^{4}$ Department of Veterinary Surgery, School of Veterinary Medicine, Tehran University, Tehran, Iran

\section{Abstract}

\section{Background}

Spinal fusions are being performed for various pathologies of the spine such as degenerative diseases, deformities, tumors and fractures. Recently, other bone substitutes such as demineralized bone matrix (DBM) have been developed for spinal fusion.

Therefore, this study was conducted to evaluate the intertransverse posterolateral fusion with the Bovine fetal growth plate (DCFGP) and compare it with commercial DBM in rat model.

\section{Methods}

A total of 16 mature male rats (aged 4 months and weighing 200-300 g) were randomly divided in two groups. After a skin incision on posterolateral site, two separate fascial incisions were made $3 \mathrm{~mm}$ from the midline. A muscle-splitting approach was used to expose the transverse processes of L4 and L5. Group I $(n=8)$ underwent with implanted Bovine fetal growth plate among decorticated transverse processes. In group II $(\mathrm{n}=8)$ commercial DBM was placed in the same manner. Fusion was evaluated by manual palpation, radiographical, gross and histopathological analysis.

\section{Results}

The manual palpation, radiological, gross and histopathological findings indicate high potential of the DCFGP in spinal fusion. At the 42nd postoperative day, new bone formation as evidenced by a bridge between L4 and L5 was visualized in all rats implanted with DCFGP and commercial DBM. The newly formed bone tissue was observed in all implanted areas on the 42nd day after operation in the two groups. 


\section{Conclusion}

The spinal fusion of the animals of both groups demonstrated more advanced osteogenic potential and resulted in proper fusion of the transverse process of lumbar vertebra.

keywords: spinal fusion, $D C F G P, D B M$, rat

Volume 8 Article 5 doi: 10.14444/1005

\section{Introduction}

Spinal fusion is indicated in patients who require segmental stabilization for mechanical instability such as those with advanced degenerative diseases, infection, deformity, tumors, congenital malformations and fractures. Spinal fusion is one of the most common procedures in spinal surgery, with more than 200,000 annual cases performed in the United States alone. ${ }^{1}$ Crucial to a successful fusion is bone graft material which is potentially osteogenic, osteoinductive (osteoinduction involves the stimulation of osteoprogenitor cells to differentiate into osteoblasts that then begin new bone formation), and osteoconductive (osteoconduction occurs when the bone graft material serves as a scaffold for new bone growth that is perpetuated by the native bone). Autograft has long been the "golden standard" bone graft material, but this is clearly associated with morbidities and limitations. In addition, despite being the optimal graft solution, autogenous graft is associated with a certain rate of pseudarthrosis, and the potential complications and morbidity from the donor site harvesting. There are many reports with different failure rates in spinal fusions with autografts that in a review article it has been showed that variation in results could be related to the scarcity of data, heterogeneity of the trials included, and some methodological defects of studies. ${ }^{6}$ For this reason, there is much interest in developing alternative bone graft materials. These would ideally help facilitating more rapid and robust fusion with less morbidity and higher success. Each potential product, however, must be established as safe and effective before entering the clinical arena. In an effort to reduce the amount of iliac graft needed, several bone substitutes have been developed. Demineralized bone matrix (DBM) is one of the bone substitutes because it has been shown that the properly demineralized cortical bone preserves the natural capacity of the native bone proteins and growth factors. ${ }^{7}$ Although the initial DBMs contained glycerol as a carrier for the DBM particles, this substance has been found to be detrimental for host cells and nephrotoxic at high doses and can migrate within the site or upon irrigation due to the water solubility of the carrier. Newer DBMs are therefore being developed with improved carrier composition and increased matrix induction concentration (the concentration of matrix that leads to osteogenesis). Studies based on the first generation DBMs are available in the literature. Advantages of DBM over other substitutes include its inherent osteoinductive capacity (unlike tricalcium phosphate and hydroxyapatite) and availability in large amounts. Presence of the growth factor $\beta$ (TGF- $\beta$ ) in the growth plate ${ }^{12}$ and bone morphogenetic proteins 2 and 7 in human and rat fetal growth plate have been identified previously. ${ }^{13}$ These proteins promote the chondroblastic differentiation of the mesenchymal cells followed by new bone synthesis by endochondral osteogenesis . For the first time Dehghani et al (2008) used segmental bovine growth plate in a long bone healing model and they showed that segmental bovine growth plate has potential osteoinductive and osteoconductive properties. ${ }^{16}$ Bigham et al 
introduced a novel biomaterial that has been prepared from demineralized calf fetal growth plate (DCFGP) that showed potential osteoinductive properties in rat submuscular model. ${ }^{17}$ To the authors' knowledge, there are no clinical studies available on the safety and efficacy of the DCFGP and additionally it has not been yet compared with a commercial DBM for spinal fusion. Therefore, the objective of the present preliminary study was to evaluate the clinical and radiological performance of a novel DCFGP used as a graft extender in posterolateral lumbar fusion in rat model.

\section{Materials and Methods}

The study was approved by the local ethics committee of our Veterinary Faculty, in accordance with the ethics standards of "Principles of Laboratory Animal Care."

\section{Animals}

Sixteen male Sprague-Dawley rats (4 months age, body weight 200-300 g) were used in this study. They were maintained in plastic cages in a room with a $12 \mathrm{~h}$-day/night cycle and an ambient temperature of $21^{\circ} \mathrm{C}$, and were allowed ad libitum access to water and standard laboratory pellets.

\section{Preparation of DCFGP}

A 6-month old bovine fetus was collected from the local slaughter house. The metacarpal bones were dissected aseptically and all soft tissue was carefully removed. Radiographs were taken to determine the growth plate's margins and limitations. With an oscillating osteotome, the proximal and distal growth plates were cut and retrieved under aseptic conditions. The retrieved growth plates were cleaned from the adjacent epiphyseal bone and primary Spongiosa and were then sliced. Demineralized materials were prepared as described by Reddi and Huggins. ${ }^{18}$ The harvested growth plate was cleaned of soft tissue and marrow, washed in sterile distilled water with continuous stirring, then washed three times in $95 \%$ ethanol for $15 \mathrm{~min}$, rinsed in ether for $15 \mathrm{~min}$, and finally was air dried overnight. The cleaned and dried growth plate was then milled (Universal Mill A-20; Tekmer Co, Cincinnati, $\mathrm{OH}$ ) to obtain $400-700 \mu \mathrm{m}$ granules and then was demineralized in $0.6 \mathrm{~N} \mathrm{HC} 1$ three times for $1 \mathrm{~h}(50 \mathrm{ml} \mathrm{HC1}$ per $\mathrm{g}$ of bone). The growth plate powder was rinsed with several changes of sterile distilled water to adjust the $\mathrm{pH}$, three times in $95 \%$ ethanol and once in ether. The growth plate powder was air dried and stored in sterile plastic containers at $4{ }^{\circ} \mathrm{C}$ until being used for implantation. This entire process was performed under sterile conditions (except for the milling) and a sample was cultured to demonstrate that the specimens contained no bacterial or fungal contamination.

\section{Surgical Technique}

All animals were anaesthetised by means of a subcutaneous injection of ketamine 100 $\mathrm{mg} / \mathrm{kg}$ and xylazine $30 \mathrm{mg} / \mathrm{kg}$. The lumbar region was shaved and surgically prepared, using three alternating betadine and alcohol washings. A posterior midline incision was made over the skin of the lumbar spine. Two longitudinal fascial incisions were made 3

$\mathrm{mm}$ lateral to the spinus processes. The L4 and L5 transverse processes were exposed and decorticated bilaterally, using a high-speed burr. 
In the animals of the DCFGP group $(\mathrm{n}=8)$ between the spaces of the transverse processes were filled with $20 \mathrm{mg}$ of DCFGP, while in those of the DBM group $(\mathrm{n}=8)$ between the space of the transverse process were filled with $20 \mathrm{mg}$ of powder form of commercial DBM (Osteotech Inc., Eatontown, NJ, USA). The fascia and skin incisions were closed with 4-0 absorbable suture. The rats were returned to their cages and allowed to eat and drink ad libitum. The rats were provided with analgesics (Buprenorphine at $0.05 \mathrm{mg} / \mathrm{kg}$ SC) and antibiotics (Enrofloxacin) for 2 days and 7 days postoperatively, respectively. The skin sutures were removed 10 days after surgery.

\section{Radiological evaluation}

Dorso-ventral radiographs were taken in 2, 4 and 6 weeks post-surgery, using a stepwedge (Aluminum Step Wedges are also used for measurement and analysis of x-ray beam quality. As in all tests, it is necessary to establish an acceptable baseline or standard for the X-ray unit's performance) to calibrate radiodensity. The implanted area was radiographed using low energy X-ray (Faxitron, Hewlett Packard, Model 43855B, McMinnville, OR) with an exposure time of $30 \mathrm{~s}(15 \mathrm{kV})$. Radio-opacity of the implanted area was scored using range from 0 (minimally opaque) to 4 (most opaque) by an investigator blinded to treatment mode.

\section{Determination of fusion by manual palpation}

At the end of the $42^{\text {nd }}$ postoperative day all animals were pharmacologically euthanized (For euthanasia an overdose of ketamin hydrochloride $100 \mathrm{mg} / \mathrm{kg}$ were used. This agent was injected intracardiac and produced sudden smooth death ${ }^{19}$ ), the spines were dissected and extracted. An independent observer blind to study assessed the spines for movement within the L4 and L5 intervertebral space by manual palpation and visualization. The spines were categorized as either fused or not fused.

\section{Gross and histopathological evaluation}

After determining the fusion criteria by manual palpation all specimens were grossly and histopathologically evaluated. At this stage the operated transverse processes were evaluated for gross signs of healing. Examination and blinded scoring of the specimens included presence of bridging bone, indicating a complete union ( +3 score), presence of cartilage ( +2 score), soft tissue or cracks within the defect indicating a possible unstable union ( +1 score), or complete instability at the defect site indicating no union ( 0 score). The implants were then removed from each animal and paraffin wax was embedded following fixation in formalin and formic acid decalcification. The sections of 5-6 $\mu \mathrm{m}$ thick (Microrn HM 340 E microtome) were stained with hematoxylin eosin (HE) and examined under a light microscope. All histopathological scores were evaluated using Emery's histopathological criteria. ${ }^{20}$

\section{Statistical analysis}

Manual palpation data were compared by chi-square evaluation. The radiological and histopathological data were compared by pair wise group comparisons Mann-Whitney U test. When the $P$-values were found to be less than 0.05 it was considered statistically significant (SPSS version 17 for windows, SPSS Inc, Chicago, USA). 


\section{Results}

\section{Manual palpation results}

The spines of the implanted rats of both DCFGP and commercial DBM groups showed bony fusion between the L4 and L5 and were not able to generate anterior-posterior or lateral movement by manual palpation at this area. There was no significant statistical difference among the two groups by chi-square evaluation $(P=1.0)$.

\section{Radiological results}

There was no intraoperative and postoperative death during the study. None of the rats sustained a wound infection or surgery complication. The radiographs did not show significant differences between the two groups at $14^{\text {th }}, 28^{\text {th }}$ and $42^{\text {nd }}$ postoperative days. The radio-opacity of the implanted area showed gradual increasing from $14^{\text {th }}$, to $42^{\text {nd }}$ postoperative days (Table 1). At the $42^{\text {nd }}$ postoperative day, new bone formation as evidenced by a bridge between L4 and L5 was visualized in all rats implanted with DCFGP and commercial DBM. An increased radio-opacity with discrete foci of densities and imparting a granular appearance to the images was observed in in the injured areas of the animals of the two groups on the $42^{\text {nd }}$ postoperative day. Radiographs also showed that there is excess bone formation at more than the single L4-5 operated level (Figure 1).

Table 1. Radiographical findings for spinal fusion at various post-operative intervals.

\begin{tabular}{|l|l|l|l|}
\hline $\begin{array}{l}\text { Postoperative } \\
\text { days }\end{array}$ & $\begin{array}{l}\text { DCFGF } \\
(\mathbf{n = 8 ;} \text { Median(Minimum- } \\
\text { Maximum) }\end{array}$ & $\begin{array}{l}\text { Commercial DBM } \\
\text { (n=8; Median(Minimum- } \\
\text { Maximum) }\end{array}$ & $\begin{array}{l}\text { P (pairwise Mann-Whitney U } \\
\text { Test) }\end{array}$ \\
\hline 14 & $3(2-4)$ & $3(2-4)$ & 0.6 \\
\hline 28 & $4(2-4)$ & $3(2-4)$ & 0.4 \\
\hline 42 & $4(3-4)$ & $4(2-4)$ & 0.1 \\
\hline
\end{tabular}



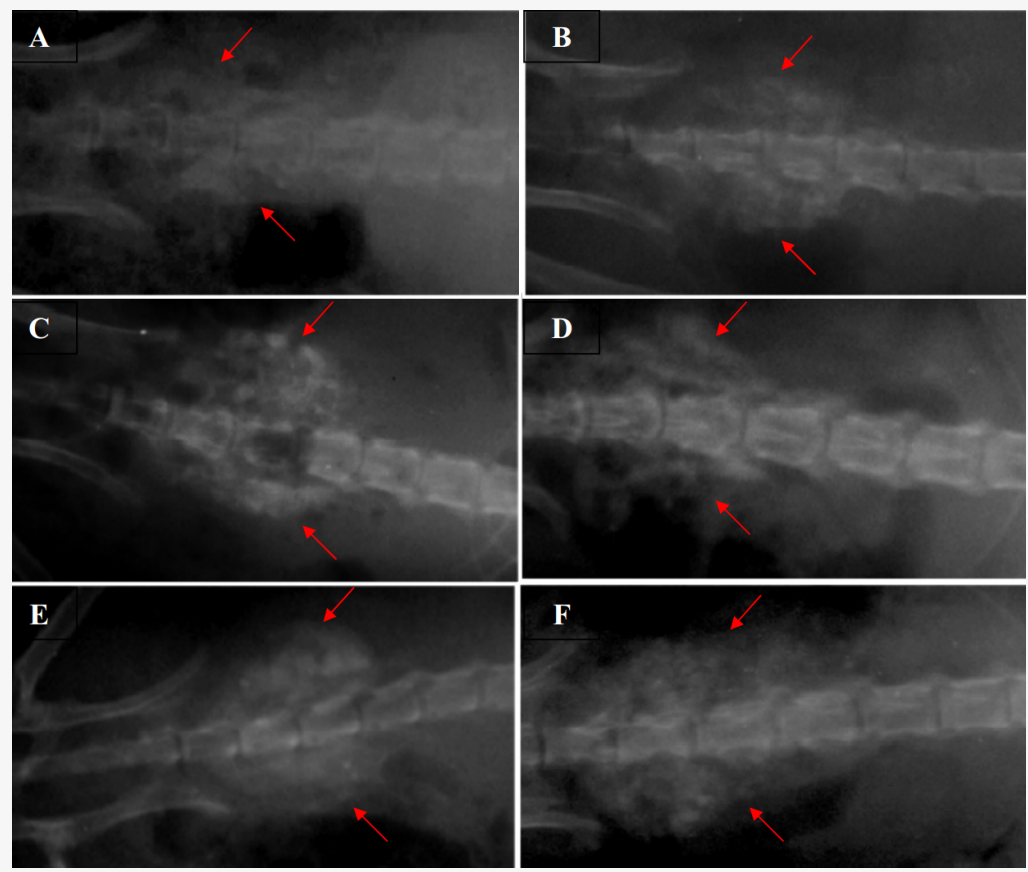

Fig. 1. Radiographic evaluation on the $14^{\text {th }}(\mathrm{A}$ and $\mathrm{B}), 28^{\text {th }}$ (C and D) and $42^{\text {nd }}(\mathrm{E}$ and $\mathrm{F})$ postoperative days. Samples of DCFGP treated spine with fusion (A, C and E) and commercial treated sample (B, D, and $\mathrm{F}$ ). The red arrows identify the radiopaque tissue masses on both sides of spine at the L4 and L5 segments.

\section{Gross and histopathological evaluation}

The implanted areas of the treated rats of both groups showed various amounts of new bone formation. The union scores of the rats administered with DCFGP and commercial DBM were not statistically significant (Table 2 ). The union scores at macroscopic level closely correlated with the radiographic union scores on day 42 post injury.

Staining of the specimens with hemotoxylin and eosin revealed that intertransverse process implantation of the DCFGP and commercial DBM lead to fibrocartilaginous response with apparent bone formation without any inflammatory response. The newly formed bone tissue was observed in all implanted areas on the $42^{\text {nd }}$ day after operation in the two groups. Bone tissue which had an optimum bone formation score was observed on the $42^{\text {nd }}$ postoperative day (Figure 1, Table 1). Differentiation of the cartilaginous tissue to a mineralized foci in this highly vascular area resulted to a bony bridge architecture and formation of an ossicle filled with bone marrow elements (day 42) (Figure 2).

Table 2. Bone production measurements at macroscopic and microscopic level.

\begin{tabular}{|l|l|l|l|}
\hline $\begin{array}{l}\text { Bone type } \\
\text { evaluation }\end{array}$ & $\begin{array}{l}\text { DCFGP }(\mathrm{n}=8 \text {; Median (Minimum- } \\
\text { Maximum) }\end{array}$ & $\begin{array}{l}\text { Commercial DBM ( } \mathrm{n}=8 \text {; Median (Minimum- } \\
\text { Maximum) }\end{array}$ & $\begin{array}{l}P \text { (Mann-Whitney U } \\
\text { test) }\end{array}$ \\
\hline Macroscopic union* & $2(2-3)$ & $2(2-3)$ & 0.6 \\
\hline
\end{tabular}


It has been shown that commercially available DBM in the surgical management of osseous defects, arthrodeses, and reconstructive procedures have been promising. In general, DBM grafts have supported healing in a timely fashion without complication and with a diminished need to harvest bone from a secondary operative site. ${ }^{22}$ Also, in an effort to augment the available grafting material as well as to increase spinal fusion rates, the utilization of a demineralized bone matrix (DBM) as a graft extender or replacement is common. ${ }^{23}$ Therefore, in the present study commercially available DBM was used as a standard control group for house made DCFGP.

Because there are many reports with failure rates of spinal fusion with autografts (as a gold standard) it is predictable that spinal fusion without any materials (as negative group) or even with autograft (as a positive group) would not be occurred at all in decorticated area therefore in the present study we had no negative nor positive groups in our study.

In this study, the spinal fusion of the animals of both groups demonstrated more advanced osteogenic potential and resulted in proper fusion of the transverse process of lumbar vertebra.

The manual palpation, radiological, gross and histopathological findings of the present study indicate a good capability in the DCFGP in spinal fusion similar to that of the commercial DBM, by the end of the 6 weeks post-surgery.

In a recent study, it has been shown that satisfactory ectopic bone formation occurred in the submuscular area of a rat model with xenogenic demineralized bovine foetal growth plate without occurring any complication. ${ }^{17}$ In addition, when the segmental calf fetal growth plate was grafted in the radial bone defect it resulted in a positive bone healing process .

Presence of transforming growth factor- $\beta$ (TGF- $\beta$ ) and bone morphogenetic proteins (BMPs 2 and 7) in the growth plate ${ }^{12}$ have been identified in human and rat fetal growth plate. ${ }^{13}$ These proteins promote chondroblastic differentiation of the mesenchymal cells, and result in new bone synthesis by endochondral osteogenesis. ${ }^{15} \mathrm{We}$ proposed that in our study, mentioned growth factors released from DCFGP in the implanted area and lead to new bone formation and also spinal fusion in rats.

The primary osteoinductive components of the demineralized bone matrix (DBM) are a series of low-molecular-weight glycoproteins including the bone morphogenetic proteins (BMPs). Decalcification of cortical bones exposes these osteoinductive growth factors buried within the mineralized matrix, thereby enhancing the bone formation process. These proteins promote the chondroblastic differentiation of the mesenchymal cells, followed with new bone synthesis by the endochondral osteogenesis . In the present study the commercial DBM had possibly this mechanism of action and lead to spinal fusion, however, in the DCFGP cases the authors propose that DCFGP, in the present study, resulted in release of the TGF- $\beta$ and BMPs 2 and 7 and these reagents were exposed to the implanted site and enhanced bone formation and lead to spinal fusion. Of course two previous study by Bae, $\mathrm{H}$. et al showed that The relative quantities of BMPs in DBMs are low, in the order of $1 \times 10(-9) \mathrm{g}$ of BMP/g of DBM. There is higher variability in concentration of BMPs among 3 different lots of the same DBM formulation than among 
different DBM formulations. This variability questions DBM products' reliability and, possibly, efficacy in providing consistent osteoinduction . In our study we did not perform any determination tests on quantity of BMPs in the DCFGP and also commercial DBM, however each two biomaterials lead to spinal fusion in rats of study.

In another study spinal fusion were reported with different rates of fusion with three different commercial DBM however there is no fusion rate with autogenous implantation in rat model. ${ }^{23}$ This finding also support our finding with DBM usage in spinal fusion.

In the present study radiographs showed that there is excess bone formation at more than the single L4-5 operated level this phenomena may be related to expanded biomaterials in the surgical sites that lead to more extended bone formation. We suggest that probably 20 $\mathrm{mg}$ of biomaterial in rat model may be too much and it should be examined in large animal model.

In the present study neither DCFGP nor commercial DBM elicited any inflammatory reaction in the grafted site. It has been reported that the demineralization process destroys the antigenic materials in bone, making DBM less immunogenic so that it does not induce immunological reaction by the host ${ }^{29}$ therefore, the authors did not observe any inflammatory reaction throughout the histopathological evaluation. In addition we performed histopathological evaluation at the end of study (42nd postoperative day) and there was no evidence of any inflammatory responses although inflammation may have been present earlier.

It seems the spinal fusion in the implanted site of the animals of the DCFGP group could be related to BMPs releasing from the demineralized grafted bovine fetal growth plate, however, the authors suggest designing further studies in this subject such as measuring at least one growth and differentiate factor to help in resolving some aspects of the mechanism of action of this product. The another missing experiment that we might perform it is biomechanical testing or immunohistochemistery staining (this would enable to better judge bone formation) that we pursue a definitive study with earlier and more evaluation points.

The results of this preliminary study indicate that satisfactory spinal fusion of the rat model occurred with the xenogenic demineralized bovine fetal growth plate (same as commercial DBM) and complications were not identified. Further studies are needed to evaluate the long-term effects of this new biomaterial on spinal fusion to document the use of this graft substitute in various clinical situations. DCFGP preparation is costeffective and readily available, in addition in clinical using of commercially available DBM (from human being) always there is awareness about affecting with transmissible infections such as HIV, Hepatitis transferring to the host however our DCFGP was made from source of calf fetal that there is not awareness about dangerous infection transfer in the host.

\section{References}

1. Boden SD. Overview of the biology of lumbar spine fusion and principles for selecting a bone graft substitute. Spine 2002;27:S26-S31. 
2. Ludwig SC, Boden SD. Osteoinductive bone graft substitutes for spinal fusion A Basic Science Summary. Orthopedic Clinics of North America 1999;30:635-645.

3. Qiu QQ, Shih MS, Stock K, et al. Evaluation of DBM/AM composite as a graft substitute for posterolateral lumbar fusion. Journal of Biomedical Materials Research Part B 2007;82:239-245.

4. Silber JS, Anderson DG, Daffner SD, et al. Donor site morbidity after anterior iliac crest bone harvest for single-level anterior cervical discectomy and fusion. Spine (Phila Pa 1976) 2003;28:134-139.

5. Younger E, Chapman M. Morbidity at bone graft donor sites. J Orthop Trauma 1998;3:192-195.

6. Lee CS, Hwang CJ, Lee DH, et al. Fusion rates of instrumented lumbar spinal arthrodesis according to surgical approach: a systematic review of randomized trials. Clin Orthop Surg 2011;3:39-47.

7. Urist MR. Bone: formation by autoinduction. Science 1965;150:893-899.

8. Bostrom MP, Yang X, Kennan M, et al. An unexpected outcome during testing of commercially available demineralized bone graft materials: how safe are the nonallograft components? . Spine 2001;26:1425-1428.

9. Wang JC, Kanim LE, Nagakawa IS, et al. Dose-dependent toxicity of a commercially available demineralized bone matrix material. Spine 2001;26:1429-1435.

10. Cammisa FPJ, Lowery G, GarWn SR, et al. Two-year fusion rate equivalency between Grafton DBM gel and autograft in posterolateral spine fusion: a prospective controlled trial employing a side-by-side comparison in the same patient. Spine 2004;29:660-666.

11. Fernyhough JC, Schimandle JJ, Weigel MC, et al. Chronic donor site pain complicating bone graft harvesting from the posterior iliac crest for spinal fusion. Spine 1992;17:1474-1480.

12. Rosier RN, O'Keefe RJ, Hicks DG. The potential role of transforming growth factor beta in fracture healing. Clinical Orthopedics Relatated Research 1998;355:S294-S300.

13. Anderson HC, Hodges PT, Aguilera XM, et al. Bone morphogenetic protein (BMP) localization in developing human and rat growth plate, metaphysis, epiphysis, and articular cartilage. Journal of Histochemistry and Cytochemistry 2000;48:1493-1502.

14. Urist MF, Sato K, Brownell AG. Human bone morphogenetic protein. Proceedings of the Society for Experimental Biology and Medicine Society for Experimental Biology and Medicine (New York, NY) 1983;194-199.

15. Urist MR, Mikulski AJ, Lietz A. Solubilized and insolubilized bone morphogenetic protein. Proceedings of the National Academy of Sciences of the United States of America 1979;76:1928-1832.

16. Dehghani SN, Bigham AS, Torabi Nezhad S, et al. Effect of bovine fetal growth plate as a new xenograft in experimental bone defect healing: radiological, histopathological and biomechanical evaluation. Cell and Tissue Banking 2008;9:91-99.

17. Bigham AS, Shadkhast M, Bigham Sadegh A, et al. Evaluation of osteoinduction properties of the demineralized bovine foetal growth plate powder as a new xenogenic biomaterial in rat. Research in Veterinary Science 2011;91:306-310

18. Reddi AH, Huggins C. Biochemical sequences in the transformation of normal fibroblasts in adolescent rats. Proceedings of the National Academy of Sciences of the United States of America 1972;1601-1605. 
19. Rigalli A, Elina Di Loreto V. Experimental surgical models in the laboratory rat. New York: Taylor \& Francis Group, 2009.

20. Emery SE, Brazinski MS, Koka A, et al. The biological and biomechanical effects of irradiation on anterior spinal bone grafts in a canine model. The Journal of Bone and Joint Surgery 1994;76:540-548.

21. Drespe IH, Polzhofer GK, Turner AS, et al. Animal models for spinal fusion. The Spine Journal 2005;5:209-216.

22. Russell JL, Block JE. Clinical utility of demineralized bone matrix for osseous defects, arthrodesis, and reconstruction: impact of processing techniques and study methodology. Orthopedics 1999;22:524.

23. Wang JC, Alanay A, Mark D, et al. A comparison of commercially available demineralized bone matrix for spinal fusion. European Spine Journal 2007;16:1233-1240.

24. Malloy KM, Hilibrand AS. Autograft versus allograft in degenerative cervical disease. Clinical Orthopaedics and Related Research 2002;394:27-38.

25. Bigham AS, Dehghani SN, Shafiei Z, et al. Experimental bone defect healing with xenogenic demineralized bone matrix and bovine fetal growth plate as a new xenograft: radiological, histopathological and biomechanical evaluation. Cell and Tissue Banking 2009;10:33-41.

26. Oshin AO, Stewart MC. The role of bone morphogenetic proteins in articular cartilage development, homeostasis and repair. Veterinary and comparative orthopaedics and traumatology 2007;20:151.

27. Bae H, Zhao L, Zhu D, et al. Variability across ten production lots of a single demineralized bone matrix product. J Bone Joint Surg Am 2010;92:427-435.

28. Bae HW, Zhao L, Kanim LE, et al. Intervariability and intravariability of bone morphogenetic proteins in commercially available demineralized bone matrix products. Spine (Phila Pa 1976) 2006;31:1299-1306; discussion 1307-1298.

29. Bauer TW, Muschler GF. Bone graft materials: An overview of the basic science. Clin Orthop Rel Res 2000;371:10-27.

\section{Corresponding author}

Dr. A. S Bigham, Department of Veterinary Surgery and Radiology, Faculty of Veterinary Medicine, Shahrekord University, Shahrekord, Iran. P. O. Box: 115. email:dr.bigham@gmail.com.

\section{Acknowledgements}

The authors would like to thank the authorities of the Veterinary School, Shahrekord University for their financial support and cooperation.

\section{Disclosures}

All authors declare no relevant financial disclosures.

Copyright (C) 2014 ISASS - International Society for the Advancement of Spine Surgery. To see more or order reprints or permissions, see http://ijssurgery.com. 